\title{
Ectopic pregnancy in the liver incidentally diagnosed by imaging: A case report
}

\author{
YE-YU CAI $^{1}$, EN-HUA XIAO ${ }^{1}$, QUAN-LIANG SHANG ${ }^{1}$ and LI-ZHI XIAO ${ }^{2}$ \\ Departments of ${ }^{1}$ Radiology and ${ }^{2}$ PET-CT, The Second Xiangya Hospital, \\ Central South University, Changsha, Hunan 410011, P.R. China
}

Received May 6, 2015; Accepted March 17, 2017

DOI: $10.3892 /$ etm.2017.4478

\begin{abstract}
The present report describes the case of a 31-year-old woman diagnosed with an ectopic pregnancy in the liver. The patient presented with amenorrhea for 40 days and abdominal distention for 27 days. A liver mass had been detected 6 days prior to presentation. Using ultrasound (US), a hyperechoic mass with a fluid sonolucent area was detected in the right hepatic lobe. Examination by computed tomography $(\mathrm{CT})$ revealed the presence of a mass in the right hepatic lobe with a slightly low-density peripheral region and an oval central portion of lower density in the plain scan; the enhanced scan revealed a significantly enhanced peripheral region and a non-enhanced central portion. ${ }^{18} \mathrm{~F}$-fluodeoxyglucose (FDG) positron emission tomography (PET)-CT showed a mass in the right hepatic lobe with an increased intake of FDG in the peripheral region (maximum standard uptake value, 5.7) and a non-increased intake of FDG in the central portion. The patient was then subjected to hysteroscopy and laparoscopy. Histopathologically, the mass was an ectopic pregnancy. The patient recovered following the surgery. In conclusion, a timely diagnosis of ectopic pregnancy was made for a 31-year-old women with an ectopic pregnancy in the liver on the basis of US, CT and PET-CT imaging results, which enabled surgery to be undertaken prior to any serious consequences. These observations may be helpful for the diagnosis of similar cases in the future.
\end{abstract}

\section{Introduction}

Ectopic pregnancy is the implantation of a fertilized egg outside the uterine cavity (1). It is a complication of the first trimester of pregnancy that arises in $1.3-2.4 \%$ of all pregnancies (1). The most common site of ectopic pregnancy is the

Correspondence to: Professor En-Hua Xiao, Department of Radiology, The Second Xiangya Hospital, Central South University, 39 Renmin Middle Road, Changsha, Hunan 410011, P.R. China E-mail: cjr.xiaoenhua@vip.163.com

Key words: liver, ectopic pregnancy, computed tomography, positron emission tomography computed tomography fallopian tube (97\% of ectopic pregnancies) (2), and the second most common is the ovary and broad ligament (3). Ectopic pregnancy may also, although rarely, occur in the liver (4), spleen (5), omentum (6), peritoneum (7), terminal ileum and colon (8).

Once an ectopic pregnancy ruptures, bleeding and other serious complications such as shock and even death (9) often ensue. If the condition is not diagnosed and treated in a timely manner, it can be life-threatening. As a common gynecological emergency, ectopic pregnancy is a focus of considerable attention by clinicians. The diagnostic means for ectopic pregnancy include clinical examination, imaging examination [for example, ultrasonography, computed tomography (CT), magnetic resonance imaging (MRI)], blood human chorionic gonadotropin (hCG) determination, blood progesterone assay and diagnostic curettage, which are often combined in clinical use $(1,2)$.

The choices of treatment for ectopic pregnancy are expectant treatment (close monitoring) (10), drug therapy (11) and surgery (1). Interventional treatment is another alternative for ectopic uterine and extra-uterine pregnancies, although non-tubal ectopic pregnancy, particularly that which is cervical, abdominal, ovarian or in a cesarean scar, represents a major clinical challenge (12).

The present case report describes the case of a 31-year-old women with an ectopic pregnancy in the liver and her US, CT and positron emission tomography (PET)-CT imaging results, from which a timely diagnosis of ectopic pregnancy was made. The study participant provided informed written consent prior to the study. The study was reviewed and approved by the Institutional Review Board of Second Xiangya Hospital, Central South University (Changsha, China).

\section{Case report}

A 31-year-old woman was admitted to the Second Xiangya Hospital, Central South University in December 2015 with the complaint of amenorrhea for 40 days, abdominal distention for 27 days. She was admitted to a local hospital 6 days earlier and a CT scan found a liver mass.

The patient had experienced one cesarean delivery in 2008 , and had undergone curettage because of embryo damage in 2009, where histopathological analysis revealed denatured villus tissue and decidual tissue. A contraceptive ring had been in place in the uterine cavity for 6 years. 
A

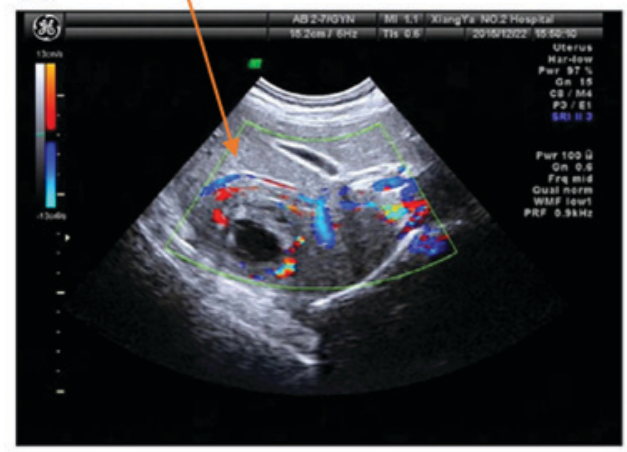

C

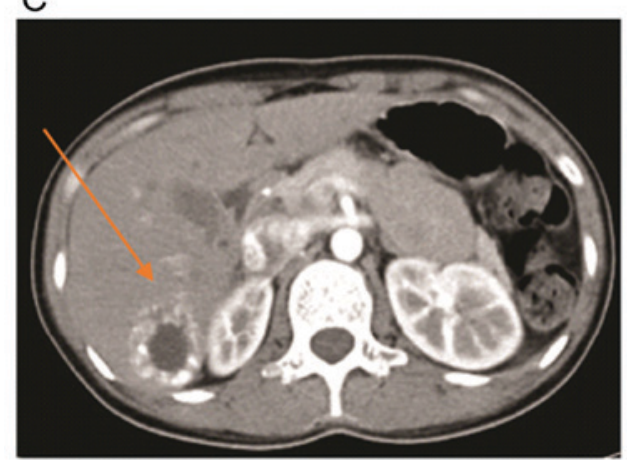

E

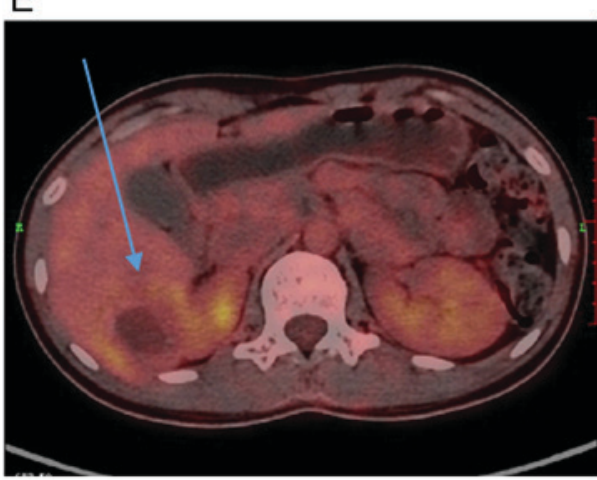

B

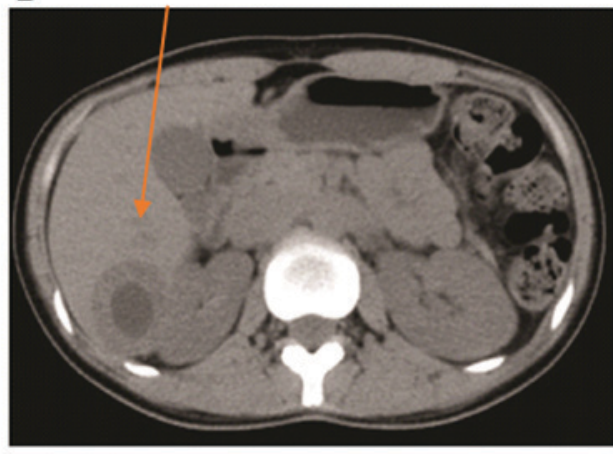

D

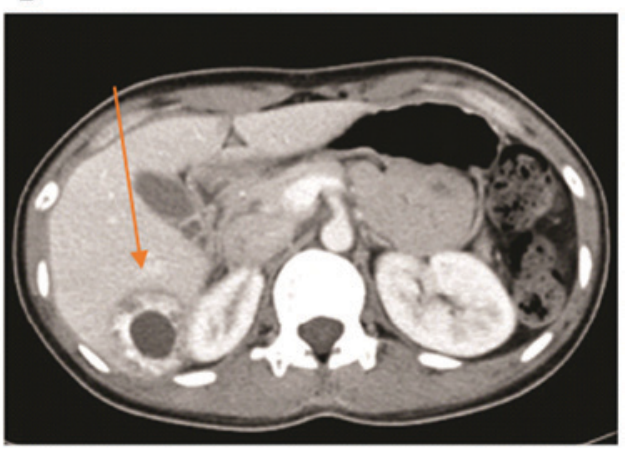

$\mathrm{F}$

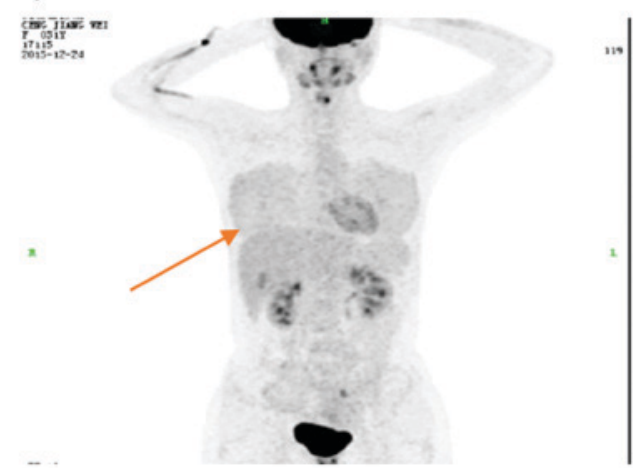

Figure 1. Imaging results for the patient with ectopic pregnancy in the liver (A) Ultrasound image. (B) CT plain scan. (C) Arterial and (D) venous phases in the enhanced CT scan. (E) Positron emission tomography-CT. (F) Positron emission tomography-CT maximum intensity projection image. Orange arrows indicate the location of the ectopic pregnancy mass. CT, computed tomography.

The patient's blood pressure was $117 / 68 \mathrm{mmHg}$ and her heart rate was 93 beats $/ \mathrm{min}$. In addition, the hemoglobin concentration was $109 \mathrm{~g} / \mathrm{l}$ (normal level $>120 \mathrm{~g} / \mathrm{l}$ ) and the serum hCG level was 49,198 U/l (normally level $<2.7 \mathrm{mlU} / \mathrm{ml}$ ) (13). Gynecological examination revealed no evident abnormalities.

An ultrasound (US) examination of the abdomen revealed no obvious gestational sac in the uterine cavity, but detected a $5.4 \times 4.6-\mathrm{cm}$ hyperechoic mass in the right hepatic lobe and a fluid sonolucent area of $2.7 \times 2.1 \mathrm{~cm}$ within the mass. The cystic sonolucent area within the fluid sonolucent area (Fig. 1A) was suggestive of an ectopic pregnancy. The intrauterine contraceptive ring was observed to be in a normal position.

CT scanning revealed a mass in the right hepatic lobe with a slightly low-density peripheral portion and oval lower-density central portion (Fig. 1B) in the plain scan, a peripheral portion with a significantly increased density in the arterial phase of the enhanced scan (Fig. 1C), a peripheral portion with a slightly increased density compared with that of the liver parenchyma in the venous phase of the enhanced scan (Fig. 1D), and a non-enhanced lower-density central portion in the enhanced scan.

${ }^{18} \mathrm{~F}-\mathrm{FDG}$ PET-CT revealed a mass in the right hepatic lobe with envelope of $4.2 \times 4.8 \mathrm{~cm}$ and a cystic hypo-dense lesion of $1.9 \times 2.7 \mathrm{~cm}$ within it. The intake of FDG was not increased in the cystic areas but that in the residual portion was increased; the maximum standard uptake value (SUVmax) was 5.7 (Fig. 1E and F).

After 3 days of preoperative preparation, hysteroscopy and laparoscopy were performed. Retro-positioning of the uterus was observed with an increased size, as would be expected for a pregnancy of $\geq 40$ days. The bilateral fallopian tubes and ovaries appeared normal, but there were some adhesions between the posterior uterine wall and the recto-uterine pouch and greater omentum. The adhesions were surgically separated. A T-type contraceptive ring placed with left part deletion in the uterine cavity was detected. There were some decidual changes of the endometrium, but no villus tissue in the uterine cavity. A curettage was undertaken with a curette 
until the suction tube sensed some roughness, and the curettage was then stopped. The right section of the liver was dissected by hepatobiliary surgeons. There was a mass of $3.0 \times 4.0 \mathrm{~cm}$ rich with blood extending out of the liver surface. Histopathologically, the mass was found to be an ectopic pregnancy. The patient was followed up for 3 months and recovered post-surgery without any more treatments.

\section{Discussion}

Ectopic pregnancies typically implant on the peritoneal surface following the partial disruption of the initial site of implantation in the fallopian tube. The pelvic cavity is the most common site, but ectopic pregnancies have been reported to occur in various sites within the peritoneal cavity (14). Primary hepatic pregnancy is an extremely rare condition with an incidence rate of 1:15,000 per inner uterus pregnancy (15). Over the past 50 years, only 21 cases have been reported in the English language medical literature, among which only $29 \%$ progressed beyond the first trimester (4,16-36).

Ectopic pregnancy is one of the common acute abdominal conditions in clinical obstetrics and gynecology (2\% of pregnancies), and comprises implantation anywhere outside the uterine cavity, including tubal pregnancy, ovarian pregnancy and abdominal cavity pregnancy (for example, mesenteric pregnancy, hepatic pregnancy, broad ligament pregnancy, cervical pregnancy, uterine rudimentary horn pregnancy and cesarean scar pregnancy); among these, the most common is tubal pregnancy, accounting for $\sim 97 \%$ of cases, while the incidence of abdominal pregnancy is $<1 \%(2,37)$.

Ectopic pregnancy in the abdominal cavity is often misdiagnosed because of its low incidence, specific location and atypical clinical manifestations and signs. Worley et al reported that only 6 of 10 women with advanced extrauterine pregnancies were discovered preoperatively, and hemorrhage was common with 9 of 10 patients requiring blood transfusions (7). Hepatic pregnancy is a rare type of abdominal cavity pregnancy. The majority of the previously reported cases of hepatic pregnancy involved liver rupture and bleeding (38) and the consequences were very dangerous. Hepatic pregnancy is frequently not diagnosed in early pregnancy and so the best treatment period is missed; the majority of patients are admitted for surgery following liver rupture. The patient in the present case soon recovered because of early diagnosis and prompt treatment.

Abdominal pregnancies usually present with acute hemoperitoneum (39) and the pre-operative diagnosis is extremely difficult. Imaging plays an important role in the diagnosis of ectopic pregnancy, particularly that of hepatic pregnancy. Although US is the primary modality used in the diagnosis of ectopic pregnancy, various forms of this condition and their complications may occasionally be further evaluated with CT or MRI. After the patient in the present case study was admitted to hospital, US, plain and enhanced CT, and PET-CT were carried out. The examinations using the three different types of imaging all prompted the diagnosis of liver ectopic pregnancy. The US scan revealed a hyper-echoic mass in the right hepatic lobe with a cystic sonolucent area within a fluid sonolucent area. In a previous case of ectopic pregnancy in the liver, Wang et al reported that US revealed only a small amount of effusion in the pelvic cavity (40). Jiang et al used a three-dimensional high-definition live rendering image to diagnosis interstitial ectopic pregnancy (41). In the present case, CT of the patient revealed a mass in the right hepatic lobe with a slightly low-density peripheral region and oval central portion with lower density in the plain scan, and a significantly enhanced peripheral portion and non-enhanced center in the enhanced scan. Wang et al reported that CT plain scanning displayed a polygonal, moderate density shadow of the left liver lobe, while enhanced CT exhibited no signs of intensification (40). Kuai et al also reported that CT transverse imaging for a patient with ectopic liver pregnancy showed a mixed density lesion within the right liver lobe under the diaphragm (42). MRI is one of the modalities typically used in the diagnosis of ectopic pregnancy. Wang et al reported that on T1-weighted imaging (WI), the lesion appeared round with a low signal intensity, while on T2WI, the lesion exhibited a high signal; with enhanced MRI, the lesion exhibited irregular mild plaque-like intensification during the venous phase (40). The PET-CT appearance of hepatic pregnancy has rarely been reported before. The present study revealed a mass in the right hepatic lobe for which the peripheral portion had increased glucose metabolism (SUVmax, 5.7) while the central portion did not exhibit increased glucose metabolism. Familiarity with the typical and atypical US, CT, MRI and PET-CT appearances of various forms of ectopic pregnancy facilitates the prompt and accurate diagnosis and treatment of this condition (43).

The risk of ectopic pregnancy has been found to be associated with previous adnexal surgery [adjusted odds ratio $(\mathrm{OR})=3.99$, 95\% confidence interval (CI): 2.40-6.63], uncertainty of previous pelvic inflammatory disease (adjusted $\mathrm{OR}=6.89$, 95\% CI: 3.29-14.41), and positive CT IgG serology (adjusted $\mathrm{OR}=5.26$, 95\% CI: 3.94-7.04); a history of infertility including tubal infertility (adjusted OR=3.62, 95\% CI: 1.52-8.63), non-tubal infertility (adjusted OR=3.34, 95\% CI: $1.60-6.93$ ) and in vitro fertilization treatment (adjusted $\mathrm{OR}=5.96,95 \% \mathrm{CI}$ : 1.68-21.21) correlated with the risk of ectopic pregnancy; and women who had previously used condoms were less likely to have an ectopic pregnancy during the current cycle (adjusted $\mathrm{OR}=0.27$, 95\% CI: 0.21-0.36) (44). The mechanism of hepatic pregnancy has not yet been clearly elucidated, but may be due to reverse tubal peristalsis causing the gestational sac to be delivered into the abdominal cavity where it plants into the upper surface of the liver through the clockwise peristalsis of the intestinal canal prior to being absorbed by the peritoneum (45). The liver has rich blood circulation, and the surface tension is not strong and is easily penetrated; thus, the early fetus grows normally, until mid-pregnancy, when liver rupture and bleeding may occur (4). Intrauterine devices (IUDs) are potentially an etiological factor. Børlum and Blom reported one case with primary hepatic pregnancy with a history of IUD use (14). The patient described in the present case report also had a history of IUD placement. Another possible cause of hepatic pregnancy has been suggested to be pelvic inflammatory disease, such as salpingitis, resulting in perihepatic adhesion (46).

Hepatic pregnancy is difficult to diagnose due to its particular location and the lack of specific clinical symptoms. The majority of cases are identified due to the bleeding caused by rupture of the liver, but by that time, it is difficult to save 
the patient. The present case did not develop bleeding or other serious consequences due to the timely diagnosis and surgery achieved through the use of imaging data. It serves as a reminder to be vigilant for patients with rare ectopic pregnancy by conducting comprehensive examinations, in order to gain valuable time for the timely application of the appropriate treatment.

In conclusion, for women of childbearing age with amenorrhea and elevated $\beta$-hCG levels, for whom US or radiographic imaging show a mixed density mass in the liver edge, but no gestational sac can be found in the uterus or by bilateral salpingo-oophorectomy, the possibility of hepatic ectopic pregnancy must be considered.

\section{Acknowledgements}

The present study was supported by the Second Xiangya Hospital, Central South University.

\section{References}

1. Taran FA, Kagan KO, Hübner M, Hoopmann M, Wallwiener D and Brucker S: The diagnosis and treatment of ectopic pregnancy. Dtsch Arztebl Int 112: 693-704, 2015.

2. Fylstra DL: Ectopic pregnancy not within the (distal) fallopian tube: Etiology, diagnosis, and treatment. Am J Obstet Gynecol 206: 289-299, 2012.

3. Goksedef BP, Kef S, Akca A, Bayik RN and Cetin A: Risk factors for rupture in tubal ectopic pregnancy: Definition of the clinical findings. Eur J Obstet Gynecol Reprod Biol 154: 96-99, 2011

4. Brouard KJ, Howard BR and Dyer RA: Hepatic pregnancy suspected at term and successful delivery of a live neonate with placental attachment to the right lobe of the liver. Obstet Gynecol 126: 207-210, 2015.

5. Zhang Y, Kang D, Zhang B, Yang L and Fan Z: Ectopic pregnancy causing splenic rupture. Am J Emerg Med 34: 1184.e1-e2, 2016.

6. Chen L, Qiu L, Diao X, Yue Q and Gong Q: CT findings of omental pregnancy: A case report. Jpn J Radiol 33: 499-502, 2015.

7. Worley KC, Hnat MD and Cunningham FG: Advanced extrauterine pregnancy: Diagnostic and therapeutic challenges. Am J Obstet Gynecol 198: 297.e1-e7, 2008.

8. Bashir RM, Montgomery EA, Gupta PK, Nauta RM, Crockett SA, Collea JV and al-Kawas FH: Massive gastrointestinal hemorrhage during pregnancy caused by ectopic decidua of the terminal ileum and colon. Am J Gastroenterol 90: 1325-1327, 1995.

9. Centers for Disease Control and Prevention (CDC): Ectopic pregnancy - United States, 1990-1992. MMWR Morb Mortal Wkly Rep 44: 46-48, 1995.

10. van Mello NM, Mol F, Hajenius PJ, Ankum WM, Mol BW, van der Veen F and van Wely M: Randomized comparison of health-related quality of life in women with ectopic pregnancy or pregnancy of unknown location treated with systemic methotrexate or expectant management. Eur J Obstet Gynecol Reprod Biol 192: 1-5, 2015.

11. Song T, Kim MK, Kim ML, Jung YW, Yun BS and Seong SJ: Single-dose versus two-dose administration of methotrexate for the treatment of ectopic pregnancy: A randomized controlled trial. Hum Reprod 31: 332-338, 2016.

12. Fornazari VA, Szejnfeld D, Elito Júnior J and Goldman SM: Interventional radiology and endovascular surgery in the treatment of ectopic pregnancies. Einstein (Sao Paulo) 13: 167-169, 2015 (In English, Portuguese).

13. Wang $\mathrm{C}$, Cheng L, Zhang $\mathrm{Z}$ and Yuan $\mathrm{Z}$ : Imaging diagnosis of hepatic ectopic pregnancy: A report of one cas. Intractable Rare Dis Res 1: 40-44, 2012.

14. Børlum KG and Blom R: Primary hepatic pregnancy. Int J Gynaecol Obstet 27: 427-429, 1988.

15. Lu JH, Yang BL and Lin QD: Liver ectopic pregnancy: A case report and analysis. Chin J Obstet Gynecol 40: 699-700, 2005.

16. Chui AK, Lo KW, Choi PC, Sung MC and Lui JW: Primary hepatic pregnancy. ANZ J Surg 71: 260-261, 2001.

17. Deladrousse E, Site O, Le Mouel A, Riethmuller D and Kastler B: Intrahepatic pregnancy: Sonography and CT findings. AJR AmJ Roentgenol 173: 1377-1378, 1999.
18. Shukla VK, Pandey S, Pandey LK, Roy SK and Vaidya MP: Primary hepatic pregnancy. Postgrad Med J 61: 831-832, 1985.

19. Kirby NG: Primary hepatic pregnancy. Br Med J 1: 296, 1969.

20. Luwuliza-Kirunda JM: Primary hepatic pregnancy. Case report. Br J Obstet Gynaecol 85: 311-313, 1978.

21. Gordeev V: Extrauterine pregnancy with fetal implantation in the liver. Klin Khir: 67-68, 1980 (In Russian).

22. Hietala SO, Andersson M and Emdin SO: Ectopic pregnancy in the liver. Report of a case and angiographic findings. Acta Chir Scand 149: 633-635, 1983.

23. Mitchell RW and Teare AJ: Primary hepatic pregnancy. A case report and review. S Afr Med J 65: 220, 1984.

24. Paulino-Netto A and Roselli A: Hepatic ectopic pregnancy: Successful surgical treatment of a patient with hepatic pregnancy and acute hemorrhage. Mt Sinai J Med 53: 514-517, 1986.

25. Veress B and Wallmander T: Primary hepatic pregnancy. Acta Obstet Gynecol Scand 66: 563-564, 1987.

26. Schlatter MF, DePree B and VanderKolk K: Hepatic abdominal pregnancy. J Reprod Med 33: 921-924, 1988.

27. Borlum KG and Blom R: Primary hepatic pregnancy. Int $J$ Gynecol Obstet 27: 427-429, 1988.

28. Harris GJ, Al-Jurf AS, Yuh WT and Abu-Yousef MM: Intrahepatic pregnancy. A unique opportunity for evaluation with sonography, computed tomography, and magnetic resonance imaging. JAMA 261: 902-904, 1989.

29. Barbosa Júnior Ade A, de Frietas LA and Mota MA: Primary pregnancy in the liver. A case report. Pathol Res Pract 187: 329-333, 1991.

30. Nichols C, Koong D, Faulkner K and Thomas G: A hepatic ectopic pregnancy treated with direct methotrexate injection. Aust N Z J Obstet Gynaecol 35: 221-223, 1995.

31. Shippey SH, Bhoola SM, Royek AB and Lonng ME: Diagnosis and management of hepatic ectopic pregnancy. Obstet Gynecol 109: 544-546, 2007.

32. Chin PS, Wee HY and Chern BS: Laparoscopic management of primary hepatic pregnancy. Aust N Z J Obstet Gynaecol 50: 95-98, 2010.

33. Qiao JC, Chang ZG, Wei JM, Liu YN, Cui HY and Zhang Y: Hepatic ectopic pregnancy treated successfully by hepatectomy. Chin Med J (Engl) 126: 4806-4807, 2013.

34. Yadav R, Raghunandan C, Agarwal S, Dhingra S and Chowdhary S: Primary hepatic pregnancy. J Emerg Trauma Shock 5: 367-369, 2012.

35. Ramphal SR, Moodley J and Rajaruthnam D: Hepatic pregnancy managed conservatively. Trop D 40: 121-122, 2010.

36. Ma J, Zhou C, Duan Z and Jiang Y: Successful management of primary hepatic pregnancy with selective hepatic artery embolization and intra-arterial methotrexate infusion. Int J Gynaecol Obstet 122: 78-79, 2013.

37. Delabrousse E, Site O, Le Mouel A, Riethmuller D and Kastler B: Intrahepatic pregnancy: Sonography and CT findings. AJR Am J Roentgenol 173: 1377-1378, 1999.

38. Faraji Darkhaneh R, Asgharnia M, Farahmand Porkar N and Alipoor AA: Predictive value of maternal serum $\beta$-hCG concentration in the ruptured tubal ectopic pregnancy. Iran J Reprod Med 13: 101-106, 2015

39. Bohiltea R, Radoi V, Tufan C, Horhoianu IA and Bohiltea C: Abdominal pregnancy-case presentation. J Med Life 8: 49-51, 2015.

40. Wang CL, Cheng XY, Yuan ZD and Wang JJ: Imaging diagnosis of ectopic pregnancy in the liver. Rare and Uncommon Diseases 14: 1-5, 2008

41. Jiang LY, Wang PH, Lee HY and Chen CY: Diagnosis of interstitial ectopic pregnancy using a three-dimensional high-definition live rendering image. Taiwan J Obstet Gynecol 54: 465-466, 2015.

42. Kuai XP, Wang SY and Qiu JM: Ectopic pregnancy implanted in the liver under the diaphragm. Taiwan J Obstet Gynecol 52: 586-587, 2013.

43. Kao LY, Scheinfeld MH, Chernyak V, Rozenblit AM, Oh S and Dym RJ: Beyond ultrasound: CT and MRI of ectopic pregnancy. AJR Am J Roentgenol 202: 904-911, 2014.

44. Li C, Meng CX, Zhao WH, Lu HQ, Shi W and Zhang J: Risk factors for ectopic pregnancy in women with planned pregnancy: A case-control study. Eur J Obstet Gynecol Reprod Biol 181: 176-182, 2014

45. Jing Nie: A case of liver pregnancy. Clin Med 21: 55-56, 2001

46. Ali V, Lilja JF, Chuang AZ, Mogallapu RV and Sabonghy E: Incidence of perihepatic adhesions in ectopic gestation. Obstet Gynecol 92: 995-998, 1998. 